\title{
Constraints on supersymmetry with light third family from LHC data
}

\author{
Nishita Desai and Biswarup Mukhopadhyaya \\ Harish-Chandra Research Institute, Chhatnag Road, Jhunsi, Allahabad - 211 019, India
}

\begin{abstract}
We present a re-interpretation of the recent ATLAS limits on supersymmetry in channels with jets (with and without b-tags) and missing energy, in the context of light third family squarks, while the first two squark families are inaccessible at the $7 \mathrm{TeV}$ run of the Large Hadron Collider (LHC). In contrast to interpretations in terms of the high-scale based constrained minimal supersymmetric standard model (CMSSM), we primarily use the low-scale parametrisation of the phenomenological MSSM (pMSSM), and translate the limits in terms of physical masses of the third family squarks. Side by side, we also investigate the limits in terms of high-scale scalar non-universality, both with and without low-mass sleptons. Our conclusion is that the limits based on zero-lepton channels are not altered by the mass-scale of sleptons, and can be considered more or less model-independent.
\end{abstract}




\section{INTRODUCTION}

The Large Hadron Collider (LHC) at CERN is now running and the two experiments ATLAS and CMS are hard at work in constraining theories beyond standard model (BSM). Supersymmetry (SUSY) [1, 2] has long been one of the most popular BSM models, due to its ability to solve the hierarchy problem and to provide a dark matter candidate in its R-parity conserving versions. The minimal supersymmetric standard model (MSSM) extends the nineteen standard model parameters to over a hundred. However, the proposed mechanisms of SUSY breaking, together with the requirements of suppresing flavour-changing neutral currents (FCNC) and obtaining the correct electroweak breaking scale often suggest a common origin of some of the parameters at high scale. This results in highly constrained models like the minimal supergravity model (mSUGRA) [3, 4] or the very similar constrained MSSM (cMSSM) [5], gauge mediated SUSY [6] and others. Alternately, the new parameters can be constrained by demanding only that all observed experimental constraints are obeyed, thus arranging SUSY breaking mass parameters in such a way as to suppress flavour-changing neutral current (FCNC) processes. This leads to a phenomenological MSSM (pMSSM) [7] which has nineteen parameters - the gaugino mass parameters $M_{1}, M_{2}$ and $M_{3}$; the squark mass parameters for the first two generations $M_{Q}, M_{U}$ and $M_{D}$, the third generation squarks $M_{3 Q}, M_{B R}, M_{T R}$; the corresponding slepton mass parameters $M_{L}, M_{R}, M_{\tau L}, M_{\tau R}$; the trilinear couplings $A_{t}, A_{b}$ and $A_{\tau}$; and the higgs sector parameters $\mu, M_{A}$ and $\tan \beta$, the ratio of the vacuum expectation values of the two Higgs doublets.

The best reach in superparticle masses at the LHC is expected in the channel with two or more hard jets and missing energy [8] which is the characteristic signature from $\tilde{g} \tilde{g}$ and $\tilde{q} \tilde{g}$ production. In particular, the simplest decays of the gluino and the squark, viz. $\tilde{g} \rightarrow q q \tilde{\chi}_{1}^{0}$ and $\tilde{q} \rightarrow q \tilde{\chi}_{1}^{0}$ result in four and three-jet states with large missing energy for the two production processes respectively. Also, the sub-dominant $\tilde{q} \tilde{q}^{*}$ and $\tilde{q} \tilde{q}$ production processes would result in two-jet final states. Therefore, looking for 2-4 jets with missing energy is known to be the best channel for SUSY searches. However, in the case that the first two generations of squarks are not accessible at the LHC (as can indeed be motivated from suppression of flavour changing neutral currents) [9, 10], the power of these searches would be dramatically reduced. Most importantly, the t-channel $\tilde{q} \tilde{g}$-type processes which contribute largest in the standard cMSSM analysis, would be severely suppressed because of the miniscule fraction of $\mathrm{b}$ and t-quarks in the proton. Similarly, other t-channel processes leading to $\tilde{q} \tilde{q}, \tilde{q} \tilde{q}^{*}$ are also suppressed. The dominant production processes are therefore $\tilde{g} \tilde{g}$ and 
s-channel $\tilde{q} \tilde{q}^{*}\left(\tilde{q}=\tilde{t}_{1,2}, \tilde{b}_{1,2}\right)$.

Since the limits on the mass of third generation squarks in a cMSSM-based analysis follow simply from limits obtained from squark and gluino production processes, they cannot be considered truly indicative of the limit on stop and sbottom masses. We therefore reinterpret the ATLAS limits in the pMSSM model where the first two generations of squarks and all sleptons are decoupled.

Considerable interest has also grown in recent times in SUGRA with non-universal high-scale masses. A high-scale parametrisation has the advantage that the masses of several particles are obtained naturally through renormalisation group (RG) running. Here too, we focus of situations where the third family sfermions are within the reach of the LHC, while the first two families are heavy. This, among other things, helps in a natural suppression of FCNC. The advantage of this scenario will be to allow us to investigate the effect of a low-mass slepton sector without requiring its full pMSSM parametrisation.

We base our study on the data from the ATLAS experiment for signatures with jets and missing energy with and without b-tagged jets [11, 13-16]. The ATLAS analysis assumes an mSUGRAtype unification for the interpretation of its data. As mentioned above, the limits from this analysis cannot be applied to the third generation squarks in a model independent way. The results have been interpreted in terms of a high-scale non-universal model in [17]. However, its dependence on mSUGRA based mass hierarchies (e.g. the lighter stop $\tilde{t}_{1}$ is always right-handed, the lightest neutralino is mostly bino-like etc.) hampers full understanding of the implication of the experimental data on the third generation squark sector. We therefore perform a more detailed study by performing a low-scale pMSSM analysis with a scan over the physical stop/sbottom masses. We also include the case of stop decay via the flavour-violating decay $\tilde{t} \rightarrow c \tilde{\chi}_{1}^{0}[18-20]$ when all other decays are forbidden by kinematics.

The phenomenology of third generation squarks has also been studied in various scenarios [2134]. It should be noted that while this manuscript was being prepared, similar studies on related issues [35, 36] have also appeared in literature.

The paper is structured as follows: in the next section, we describe our simulation strategy and demonstrate the degree of agreement with the original ATLAS analysis. In section III we describe the phenomenological modelling of the stop and sbottom sectors and present the results corresponding to this scenario. The case of various high-scale non-universal scenarios is described in section IV. We present our conclusions in section $\mathrm{V}$. 


\section{CMSSM: SIMULATION OF THE SIGNAL AND ATLAS EXCLUSION CURVES}

We simulate the signal using PYTHIA 6.4 [37] and all strong production cross sections are normalised to their next-to-leading order (NLO) values as obtained from Prospino 2.1 [38]. The renormalisation and factorisation scales are set to the average of masses in the final state of the hard scattering process. We follow the detector acceptance region for all reconstructed objects and apply all the cuts as described in the ATLAS papers.

The full set of identification and acceptance cuts is as follows:

- Electrons: (1) $p_{T}>20 \mathrm{GeV}$ (2) $|\eta|<2.47$ (3) Sum of $p_{T}$ of particles within $\Delta R=$ $\sqrt{(\Delta \eta)^{2}+(\Delta \phi)^{2}}<0.2$ should be less than $10 \mathrm{GeV}$ (4) Event vetoed if electron found in $1.37<|\eta|<1.52$.

- Muons: (1) $p_{T}>20 \mathrm{GeV}$ (2) $|\eta|<2.4$ (3) Sum of $p_{T}$ of charged tracks within $\Delta R=$ $\sqrt{(\Delta \eta)^{2}+(\Delta \phi)^{2}}<0.2$ should be less than $1.8 \mathrm{GeV}$.

- Jets: (1) Formed using Anti-kt algorithm from Fastjet with parameter $R=0.4$ (2) $p_{T}>20$ $\mathrm{GeV}$.

- b-tagged jets: A jet is b-tagged if a b-hadron falls within a cone of radius $R$ from a jet. We have checked that this reproduced the $50 \%$ tagging efficiency for $t \bar{t}$ samples as mentioned in [16].

- Missing transverse energy is calculated by summing over the $p_{T}$ of all objects and all stable visible particles not belonging to any reconstructed objects but falling within $|\eta|<4.9$ with $p_{T}>0.5 \mathrm{GeV}$.

To account for detector effects, we smear the momenta of leptons and jets obtained from the Monte Carlo generator according to

$$
\frac{\sigma(E)}{E}=\frac{a}{\sqrt{E}} \oplus b
$$

The values of $a$ and $b$ are $(0.11,0.007)$ for electrons, $(0.03,0.06)$ for muons and $(1.0,0.05)$ for jets [52]. After smearing, we apply cuts used in each ATLAS analysis under consideration.

A cross-check of our analysis is the correct reproduction of the missing transverse energy (MET) and effective mass $\left(M_{\text {eff }}\right)$ distributions and consequently the reproduction of the ATLAS 
exclusion curves [13, 14] in the context of mSUGRA. The online resources for the jets+MET analysis for $35 \mathrm{pb}^{-1}[11]$ provide both the benchmark points used in the scan as well as the efficiencies and cross sections at each of the points. We use this information to verify the correctness of our simulation. We present in Fig. 1, the final exclusion curves for the jets+MET analysis at $165 \mathrm{pb}^{-1}$ which are obtained using the same acceptance and smearing parameters. We find that the "true" ATLAS curve lies in between our leading order (LO) and NLO curves in all cases.

Since we aim to determine the limits on third generation squarks, the limits on b-tagged events are of particular importance and are expected to provide much stronger limits. Therefore, reliable modelling of b-tagging is of prime importance in looking at these signals. The work reporting the analysis of b-jet+MET describes the performance of the b-tagging algorithm as having an efficiency factor of $50 \%$ for a $t \bar{t}$ sample. We reproduce this number by this simple algorithm: we first form jets using the anti- $k_{T}$ algorithm using FASTJET 2.4 [39] with the radius parameter $R=0.4$. A jet is assumed to be b-tagged if a b-hadron is found within a distance $R$ from its axis. Since the correct reproduction of MET and $M_{\text {eff }}$ has been verified from the non-b-tagged samples, we can see that this algorithm gives a reasonably correct b-jet tagging by looking at the bottomright panel in Fig. 1. Here too, we find that the LO and NLO curves encapsulate the ATLAS curve reliably.

In all the above cases, we find that the LO curve only slightly underestimates the ATLAS limits. In the worst case, the difference between the LO limits and the ATLAS curve is within $20 \%$. We therefore mostly present the LO mass limits in the subsequent study. Our LO results with simplistic detector simulation do not differ by more than $20 \%$ from what a full re-analysis of the data, including detector responses, would give. Our cross-checks on the mSUGRA results convince us such limits are adequate for putting across our main point, given the uncertainty of detector effects.

We use the results on jets+MET for $1.04 \mathrm{fb}^{-1}$ [15] and b-jets+MET results for $0.83 \mathrm{fb}^{-1}[16]$ for our analysis. As mentioned above, our simulation includes all the cuts in each of the channels under consideration. The benchmark points for our analyses are obtained using SUSPect 2.41 [40]. To obtain the exclusion curves, we use the value of cross section times acceptance provided by the ATLAS analysis. The values corresponding to different channels taken from these ATLAS analyses are summarised in Table I. The names of the signal regions are the same as those used in the respective ATLAS analyses.

We concentrate here only on signals without leptons in the final state. The primary reason for 

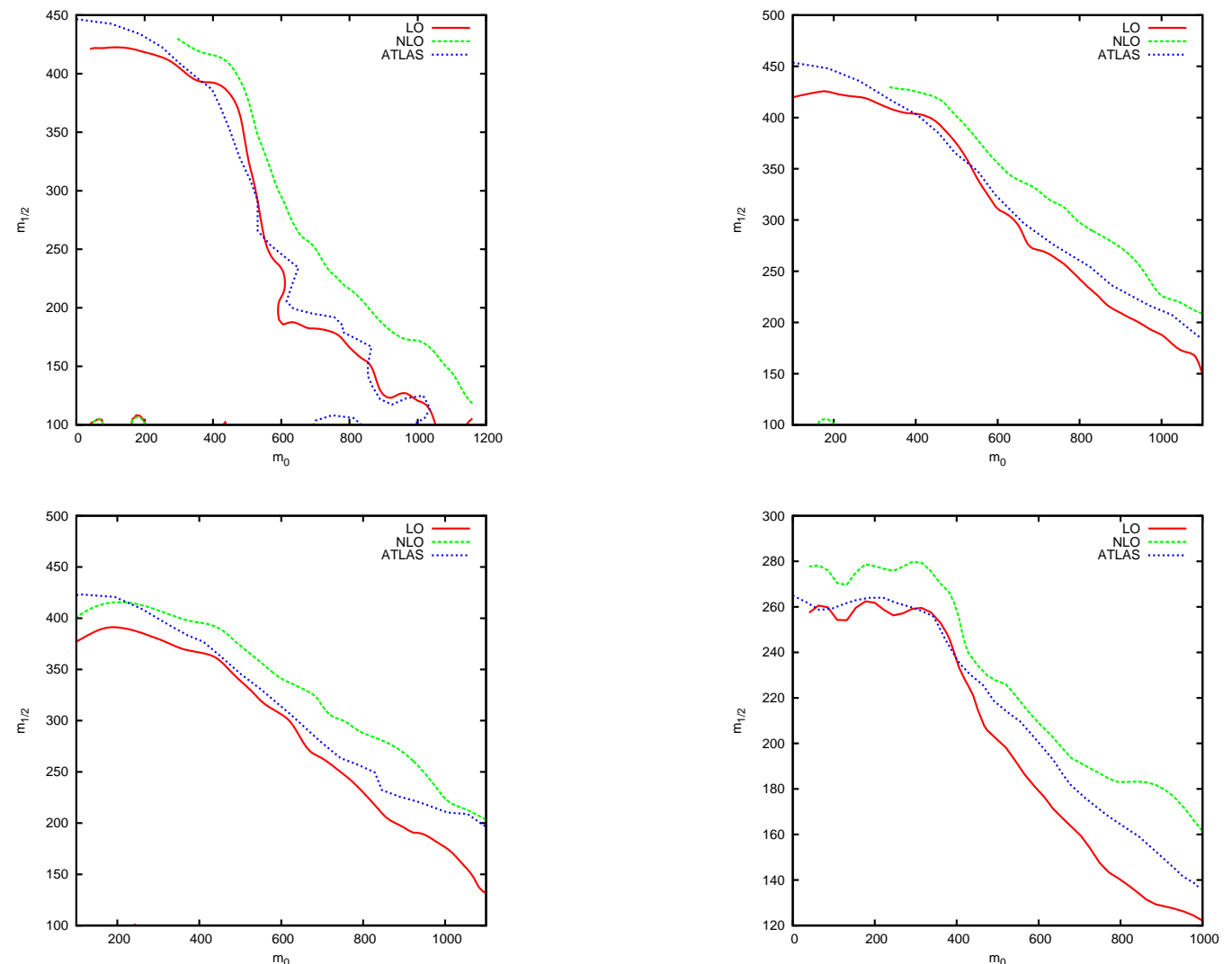

FIG. 1: Comparison of our exclusion curves with the ATLAS exclusion curves [13, 14]. The panels represent: 2-jet with $165 \mathrm{pb}^{-1}$ (top-left), 3-jet with $165 \mathrm{pb}^{-1}$ (top-right), 4-jet with $165 \mathrm{pb}^{-1}$ (bottom-left) and b-jet with 0-leptons with $35 \mathrm{pb}^{-1}$ (bottom-right). The true ATLAS curve lies between our LO and NLO contours in all cases.

this is that we wish to investigate the third generation squark sector in as model independent a way as possible. Leptonic states generally result from decays on gauginos into gauge bosons or into sleptons which then decay into leptons. For a pMSSM study based on leptonic signatures, it would therefore be imperative to also include a completely general gaugino sector as well as a low-mass slepton sector. Since adding a completely phenomenological slepton sector means adding five new parameters which complicate the analysis beyond too much, our low-scale analysis deals with a decoupled sleptonic sector. It is, in principle, possible that the limits obtained in the decoupled slepton limit are diluted when decays into sleptons (and hence leptons) are possible. We include the possibility of a low-mass slepton sector in section IV, where we use high-scale parametrisation and allow the RG running to determine the masses in the slepton sector. However, we shall see that 
allowing low-mass sleptons do not make significant difference to the limits for signatures based on jets and missing energy.

We perform our analysis retaining the cMSSM-like gaugino mass pattern $M_{1}: M_{2}: M_{3}=1$ : $2: 6$. In general, for most gaugino mass patterns, we expect our results to remain fairly unchanged since our signal does not depend strongly on particles obtained from intermediate decays in SUSY cascades. However, we explicitly comment at the end of the paper on the extreme cases of gaugino mass patterns that would be likely yield results drastically different from ours.

It is also possible to ask what fraction of the pMSSM phase space is ruled out by current data. For the effect of the experimental limits on the full pMSSM space, we refer the reader to [41, 42]. The effects on the cMSSM parameter space are addressed in, for example, [42_-45] whereas other interpretations of the recent LHC data on SUSY have been discussed in [46-49].

\begin{tabular}{lc}
\hline Channel & $\sigma \times a c c(\mathrm{fb})$ \\
\hline 2 jets + MET & 24 \\
3 jets + MET & 30 \\
4 jets + MET $\left(M_{\text {eff }}=1 \mathrm{TeV}\right)$ & 32 \\
\hline 1 btag $+M_{\text {eff }}>500(3 \mathrm{JA})$ & 288 \\
1 btag $+M_{\text {eff }}>700(3 \mathrm{JB})$ & 61 \\
2 btag $+M_{\text {eff }}>500(3 \mathrm{JC})$ & 78 \\
2 btag $+M_{\text {eff }}>700(3 \mathrm{JD})$ & 17 \\
\hline
\end{tabular}

TABLE I: The values of cross section times acceptance from ATLAS analysis used for applying exclusion limits. The first set uses $1.04 \mathrm{fb}^{-1}$ of data [15] whereas the second uses $0.833 \mathrm{fb}^{-1}$ of data [16].

\section{PARAMETERISATION OF THE STOP AND SBOTTOM SECTOR}

We work in the pMSSM framework where the parameters are assigned at the low-scale. The program SUSPECT is used to ensure that electroweak symmetry breaking has correctly taken place and the spectrum is consistent. To start with, we retain the cMSSM-like gaugino mass ratios, correspond to $M_{1}: M_{2}: M_{3} \simeq 1: 2: 6$ among the $\mathrm{U}(1), \mathrm{SU}(2)$ and $\mathrm{SU}(3)$ gaugino masses. The squark masses of the first two generations and all the slepton masses are set to $2 \mathrm{TeV}$ which is beyond the reach of the $7 \mathrm{TeV}$ LHC run. The stop and sbottom sector can each be described 
by three parameters - the two mass eigenstates and the mixing angle. We use these as the input parameters for the scan and use the diagonalisation to determine the left and right handed mass parameters of the pMSSM. The stop sector requires three parameters - the masses $M_{\tilde{t}_{1}}$ and $M_{\tilde{t}_{2}}$ and the stop mixing angle $\theta_{\tilde{t}}$. Using the stop mass-squared matrix diagonalisation condition

$$
\left(\begin{array}{cc}
M_{\tilde{t}_{1}}^{2} & 0 \\
0 & M_{\tilde{t}_{2}}^{2}
\end{array}\right)=\mathcal{R}\left(\begin{array}{cc}
M_{3 Q}^{2} & m_{t} X_{t} \\
m_{t} X_{t} & M_{T R}^{2}
\end{array}\right) \mathcal{R}^{-1} ; \mathcal{R}=\left(\begin{array}{cc}
\cos \theta_{\tilde{t}} & \sin \theta_{\tilde{t}} \\
-\sin \theta_{\tilde{t}} & \cos \theta_{\tilde{t}}
\end{array}\right)
$$

where $X_{t}=A_{t}-\mu \cot \beta$, we can use the low-scale masses $M_{\tilde{t}_{1}}, M_{\tilde{t}_{2}}$ and $\theta_{\tilde{t}}$ as the input parameters which uniquely determine $A_{t}$ given $\mu$ and $\tan \beta$. The left handed sbottom mass is expected to be close to the left handed stop mass since they are derived from the same parameter $\left(M_{3 Q}\right)$ in the low-scale pMSSM model. The right handed sbottom mass and $A_{b}$ can then be set depending on the requirement of $M_{\tilde{b}_{1}}, M_{\tilde{b}_{2}}$ and $\theta_{\tilde{b}}$.

Assuming that the third generation squarks and the gluino are the only strongly charged superparticles accessible at the LHC, we investigate in particular, the following cases:

- Case A: $\sin \theta_{\tilde{t}}=0.99$ i.e. $\tilde{t}_{1} \simeq \tilde{t}_{R}$ is the lightest squark. This is commonly the case in cMSSM models. We also set $M_{\tilde{b}_{1}} \simeq M_{\tilde{t}_{2}} \simeq M_{\tilde{b}_{2}}=M_{\tilde{t}_{1}}+500 \mathrm{GeV}$, which makes the sbottom sector somewhat heavier than the lighter stop. We scan the parameter space in $M=M_{\tilde{t}_{1}}$.

- Case B: $\sin \theta_{\tilde{t}}=0.01$ i.e. $\tilde{t}_{1} \simeq t_{L}, \simeq \tilde{b}_{1} \simeq \tilde{b}_{L}$. The lightest stop and sbottom are nearly degenerate and mostly left handed. $M_{\tilde{t}_{2}} \simeq M_{\tilde{b}_{2}}=M_{\tilde{t}_{1}}+500 \mathrm{GeV}$. We scan the parameter space in $M=M_{\tilde{t}_{1}}=M_{\tilde{b}_{1}}$.

- Case C: $\tilde{b}_{1} \simeq \tilde{b}_{R}$ is the lightest squark. $M_{\tilde{b}_{2}} \simeq M_{\tilde{t}_{1}} \simeq M_{\tilde{t}_{2}}=M_{\tilde{b}_{1}}+500 \mathrm{GeV}$ with $\sin \theta_{\tilde{t}}=0.70$ and $\sin \theta_{\tilde{b}}=0.99$. We scan the parameter space in $M=M_{\tilde{b}_{1}}$.

- Case D: $M_{\tilde{t}_{1}} \simeq M_{\tilde{b}_{1}} \simeq M_{\tilde{t}_{2}} \simeq M_{\tilde{b}_{2}}$. This is the case of maximal mixing in both stop and sbottom sectors. The most stringent limits on the light third family scenario will arise for this particular case, as it allows all four squarks to be produced with similar cross sections. The scan in this case is over the common mass of all the third generation squarks.

In each case, we are now able to perform a scan over the $M-M_{2}$ plane, where $M$ is the mass of $\tilde{t}_{1}$ in cases $\mathrm{A}, \mathrm{B}$ and $\mathrm{D}$ and $\tilde{b}_{1}$ in the case $\mathrm{C}$. The Tevatron reach for searches in the $\tilde{t}_{1} \rightarrow c \tilde{\chi}_{1}^{0}$ rule out stop masses up to $180 \mathrm{GeV}$ [50]. We therefore start our search at $M_{\tilde{t}_{1}}=200 \mathrm{GeV}$ and scan up 


\begin{tabular}{lc}
\hline Parameter & Scan range \\
\hline$M_{\tilde{t}_{1}}$ & $100-2000 \mathrm{GeV}$ \\
$M_{2}$ & $150-600 \mathrm{GeV}$ \\
$\tan \beta$ & $5,10,40$ \\
$\mu$ & $-200,200,500,1000 \mathrm{GeV}$ \\
\hline
\end{tabular}

TABLE II: The parameters of the scan in $M_{\tilde{t}_{1}}-M_{2}$ space.

to $2 \mathrm{TeV}$. Also, since we assume gaugino mass unification, we use the chargino mass limits from Tevatron $\left(M_{\tilde{\chi}_{1}^{+}}>164 \mathrm{GeV}\right)$ to start our scan at $M_{2}=150 \mathrm{GeV}$ and vary $M_{2}$ up to a value of 600 $\mathrm{GeV}$ which would correspond to gluino mass of $1.8 \mathrm{TeV}$ and therefore cover the entire range of masses reachable at the $7 \mathrm{TeV}$ run of the LHC. We fix $M_{A}=400 \mathrm{GeV}$ and perform this scan for 12 combinations of 3 values of $\tan \beta$ and 4 values of $\mu$, which are listed in Table

The hierarchy among $M_{1}, M_{2}$ and $\mu$ determines the composition of the neutralino and chargino sector and therefore has strong effects on the limits. In particular, for cases with low values of $\mu$, the lightest neutralino has a significant higgsino fraction. When $M_{2}$ becomes large enough, the states $\tilde{\chi}_{1}^{0}$ and $\tilde{\chi}_{1}^{+}$become higgsino dominated and their masses remain close to the value of $\mu$. In this case, the masses and compositions of $\tilde{\chi}_{1}^{0}, \tilde{\chi}_{1}^{+} \tilde{\chi}_{2}^{0}$ are not affected by changes in the value of $M_{2}$, so long it is considerably larger than $\mu$. For large $\mu$, on the other hand, the allowed decays of the squark will depend strongly on $M_{2}$ up to very large values. Therefore, we expect that for low squark masses and $\mu$ low with respect to $M_{2}$, the exclusion contour is relatively insensitive to $M_{2}$. This can clearly be seen in figure 2 and best illustrated in the fourth panel corresponding to Case-D. Here, the production cross section is high because all four third generation squarks are degenerate. Moreover, it can clearly be seen that $\mu=-200$ and 200 both lead to large exclusion in $M_{2}$ for small masses. The effect is similar also in the panel corresponding to Case-B. The third panel, corresponding to sbottom being the lightest shows minimal change with changing $\mu$. This is mostly because the decay $\tilde{b}_{1} \rightarrow b \tilde{\chi}_{1}^{0}$ is always open irrespective of $\mu$ due to the small mass of the b-quark. Thus, the sensitivity to $\mu$ is reduced.

The channel with two b-tagged jets and high missing energy (called 3JD in [16]) results in most stringent limits in all cases. The cross section ruled out in this case $(0.017 \mathrm{pb})$ is similar to the the number for 2 jets $+\operatorname{MET}(0.024 \mathrm{pb})$, however, the latter does not contribute significantly to the parameter space ruled out. The reason for this is two-fold: first, the effective mass cut of 1 

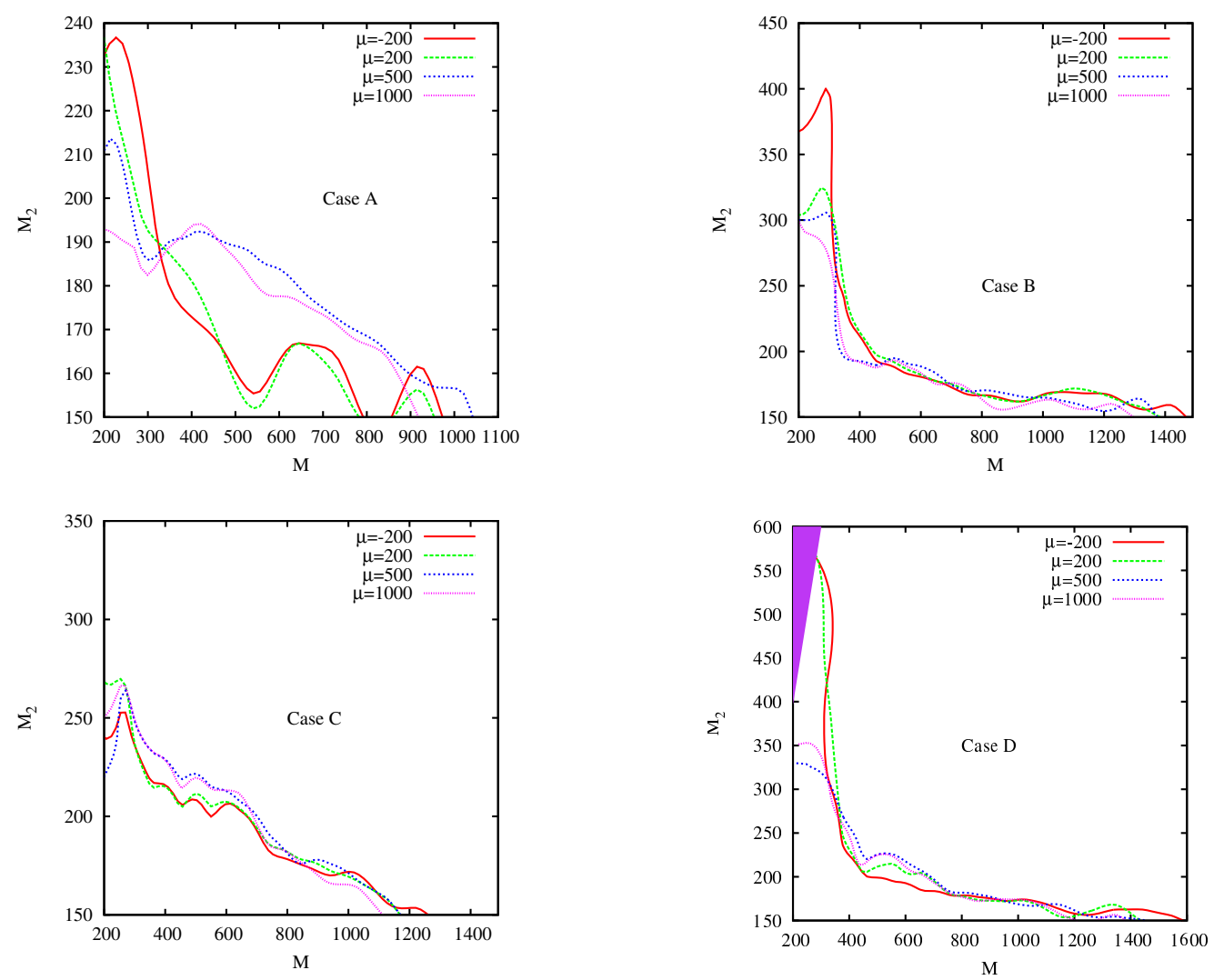

FIG. 2: The dependence of the exclusion curves on different values of $\mu$ for $\tan \beta=10$. The least dependence is for the case where the sbottom is the lighter squark. The shaded region at the top in the fourth panel corresponds to stop LSP and is therefore ruled out. The x-axis refers to $M_{\tilde{t}_{1}}$ for Cases A, B and D and $M_{\tilde{b}_{1}}$ for Case C.

$\mathrm{TeV}$ for the jets+MET final states has a much smaller efficiency for small stop masses. Secondly, the missing energy cut for 3JD is a smaller, fixed number $(130 \mathrm{GeV})$ as compared to the $M_{\text {eff }}$ dependent one $\left(\mathrm{MET}>0.3 M_{\text {eff }}\right)$ in the 2 jets + MET case. The reduction of effeciency when demanding 2 b-tagged jets is not very severe in $\tilde{g} \tilde{g}$ production events due to four b-quarks in the final state. Even in the regions where exclusion is dominated by $\tilde{q} \tilde{q}^{*}$-type production, we still find that the 3JD channel results in the best exclusions.

To estimate the effect of enhancement due to NLO corrections in the production cross section, we present the comparison of LO and NLO curves for each case with $\tan \beta=10$ and $\mu=500 \mathrm{GeV}$ in Fig. 3. The large k-factors $(\sim 2.5)$ in most of the parameter space result in much stronger limits from the NLO curves. However, taking note of the results of our cMSSM limits in Section $\amalg$, where the LO limits are closer to actual ATLAS limits, we take the conservative approach of 

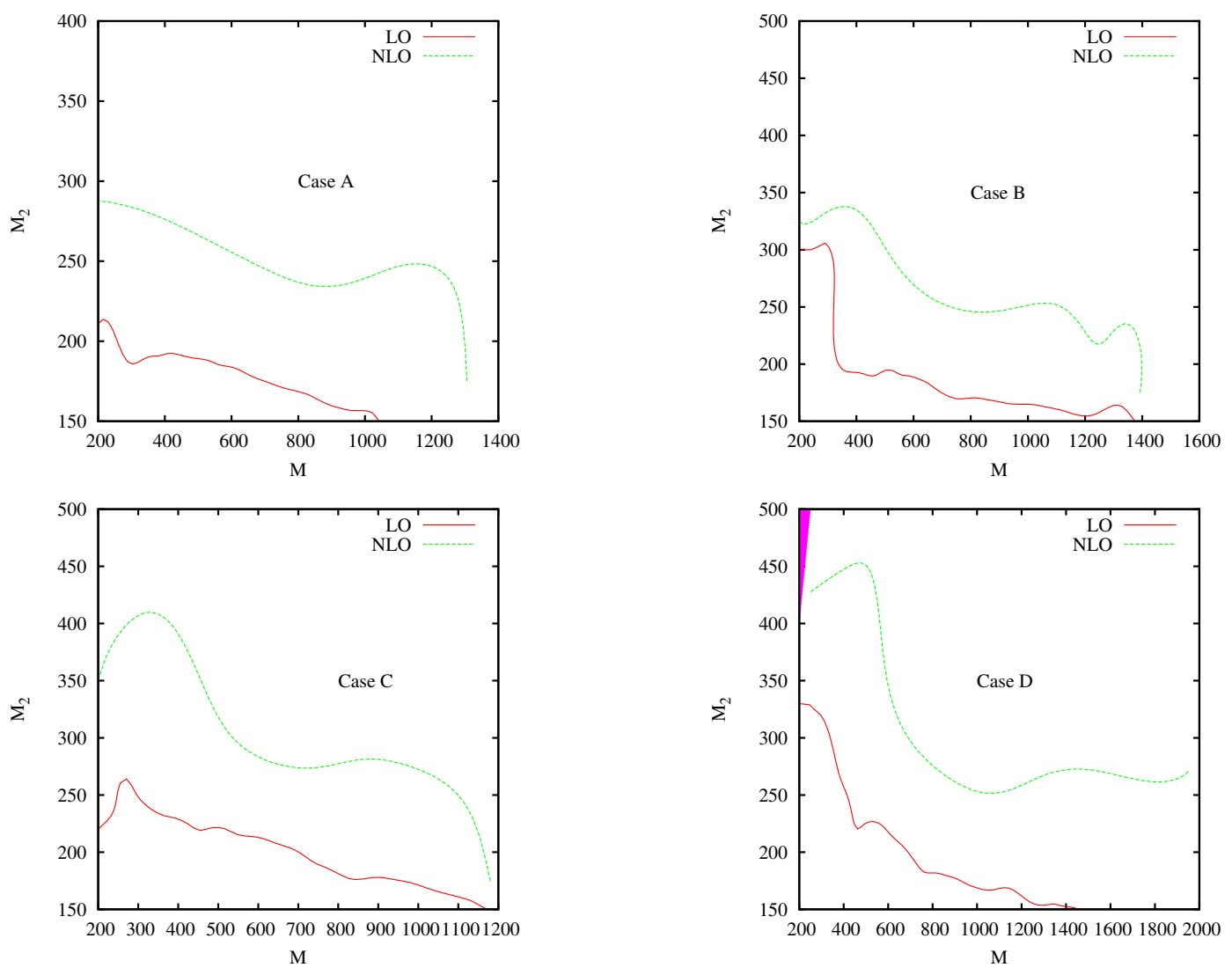

FIG. 3: Comparison of LO and NLO exclusion curves for Cases A, B, C and D, illustrated for values $\tan \beta=10$ and $\mu=500 \mathrm{GeV}$. The $\mathrm{x}$-axis refers to mass of the lightest third generation quark in each case, as discussed in the text.

presenting LO limits for our study.

\section{Results: Case A}

This is the case closest to mSUGRA-type models where $\tilde{t}_{1}$ is the lightest squark. The primary production processes in this case are $\tilde{g} \tilde{g}$ and $\tilde{t}_{1} \tilde{t}_{1}^{*}$. Since our scan starts with $M_{2}=150 \mathrm{GeV}$, i.e. a gluino mass of $450 \mathrm{GeV}$, the decay $\tilde{g} \rightarrow t \bar{t} \tilde{\chi}_{1}^{0}$ is always open and forms the dominant decay mode. In the low mass regions which are probed by the LHC data, the dominant decay mode of the $\tilde{t}_{1}$ depends on the mass hierarchy of $\tilde{t}_{1}, \tilde{\chi}_{1}^{0}$ and $\tilde{\chi}_{1}^{+}$. Since we are working in R-parity conserving models, we disallow the region where the lighter stop is the lightest supersymmetric particle (LSP) i.e. the region where $M_{\tilde{t}_{1}}<M_{\tilde{\chi}_{1}^{0}}$. Following this, the hierarchy $M_{\tilde{\chi}_{1}^{0}}<M_{\tilde{t}_{1}}<M_{\tilde{\chi}_{1}^{+}}$results in the case of stop NLSP (next-to-LSP). The dominant mode is $\tilde{t}_{1} \rightarrow t \tilde{\chi}_{1}^{0}$ if $M_{\tilde{t}_{1}}>M_{t}+M_{\tilde{\chi}_{1}^{0}}$. 

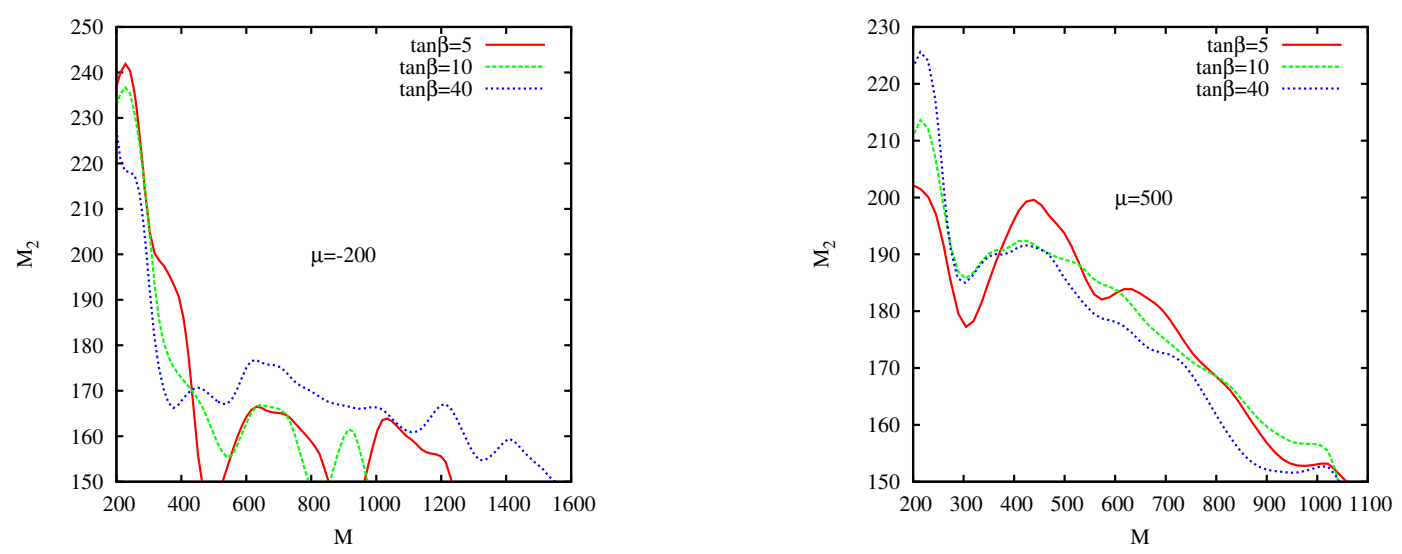

FIG. 4: Exclusion curves for Case-A (stop lightest scenario). The x-axis refers to the mass of $\tilde{t}_{1}$ squark.

Otherwise, we expect $\tilde{t}_{1}$ to decay via three-or four-body decays or via the mode $\tilde{t}_{1} \rightarrow c \tilde{\chi}_{1}^{0}$. For this work, we assume that this last mode dominates over the three or four body decays. Finally, in the case where $M_{\tilde{t}_{1}}>M_{b}+M_{\tilde{\chi}_{1}^{+}}$, the decay into $b \tilde{\chi}_{1}^{+}$is also open. For larger stop masses, decays into other neutralinos, second chargino or gluino may also open.

The effect of various values of $\tan \beta$ for two values of $\mu$ are shown in Fig. 4. The exclusion curves show minor dependence on $\tan \beta$. We find that for low values of $\mu$, low $\tan \beta$ results in a larger reach in $M_{2}$ whereas high $\tan \beta$ results in a larger reach in $M_{\tilde{t}}$. This trend is reversed for large values of $\mu$, as can be seen in the panel corresponding to $\mu=500 \mathrm{GeV}$ of Fig. 4. However, it must be reiterated, that this variation cannot be considered experimentally significant due to the uncertainties on the exclusion curves in our analysis.

\section{Results: Case B}

In this case, the $\tilde{t}_{1}$ and $\tilde{b}_{1}$ form a degenerate pair of lightest squarks. They are both primarily left handed and therefore have an enhanced coupling to wino-like states. Again, due to the requirement of neutral dark matter candidate, we disallow any region with stop or sbottom LSP. The decays of the lighter stop are similar to those in case A. The decay of the lighter sbottom into $b \tilde{\chi}_{1}^{0}$ is almost always allowed and will form the dominant decay for most of the low mass region. In cases of large $\mu$, where $M_{\tilde{\chi}_{2}^{0}} \simeq M_{2}$, the decay $\tilde{b}_{1} \rightarrow b \tilde{\chi}_{2}^{0}$ will dominate over $\tilde{b}_{1} \rightarrow b \tilde{\chi}_{1}^{0}$ and similarly for stop decays. The decay $\tilde{b}_{1} \rightarrow t \tilde{\chi}_{1}^{+}$is relatively disfavoured due to large top mass. The gluino decays dominantly to $b \bar{b} \tilde{\chi}_{1}^{0}$ in the region $M_{\tilde{g}}<M_{\tilde{b}_{1}}$ and to $b \tilde{b}_{1}$ otherwise. The decays to corresponding top-sector are again disfavoured due to large top mass. 

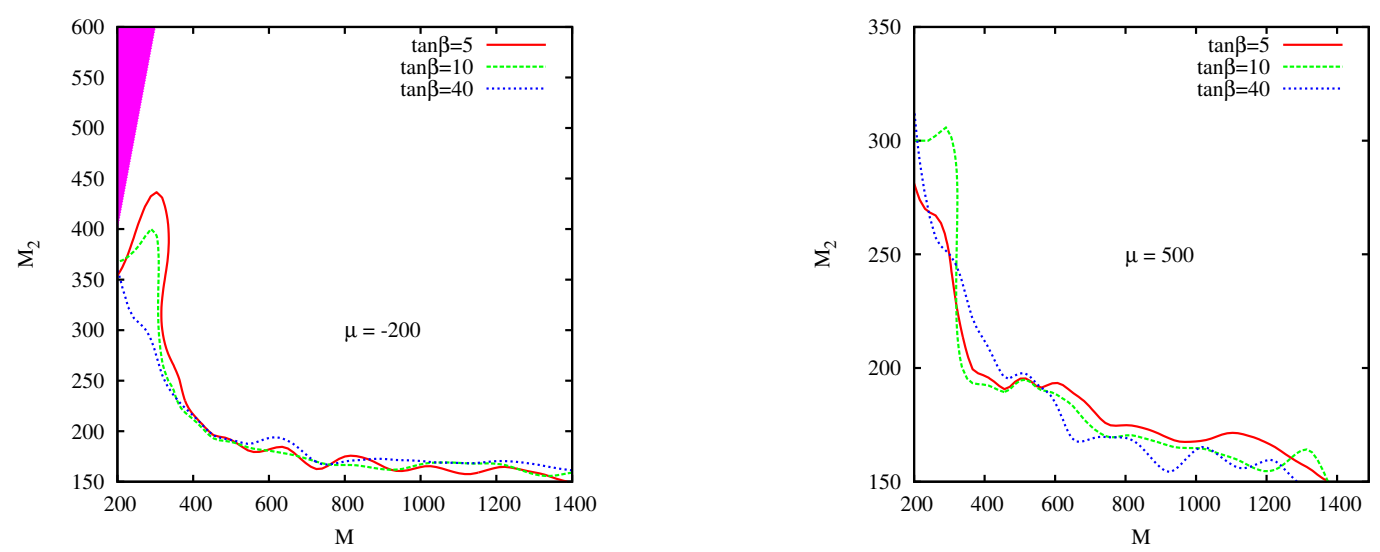

FIG. 5: Exclusion curves for Case-B (left-handed degenerate stop and sbottom scenario). The x-axis refers to the common mass of $\tilde{b}_{1}$ and $\tilde{t}_{1}$ squarks. The shaded region at the top in the fourth panel corresponds to stop/sbottom LSP and is therefore ruled out.
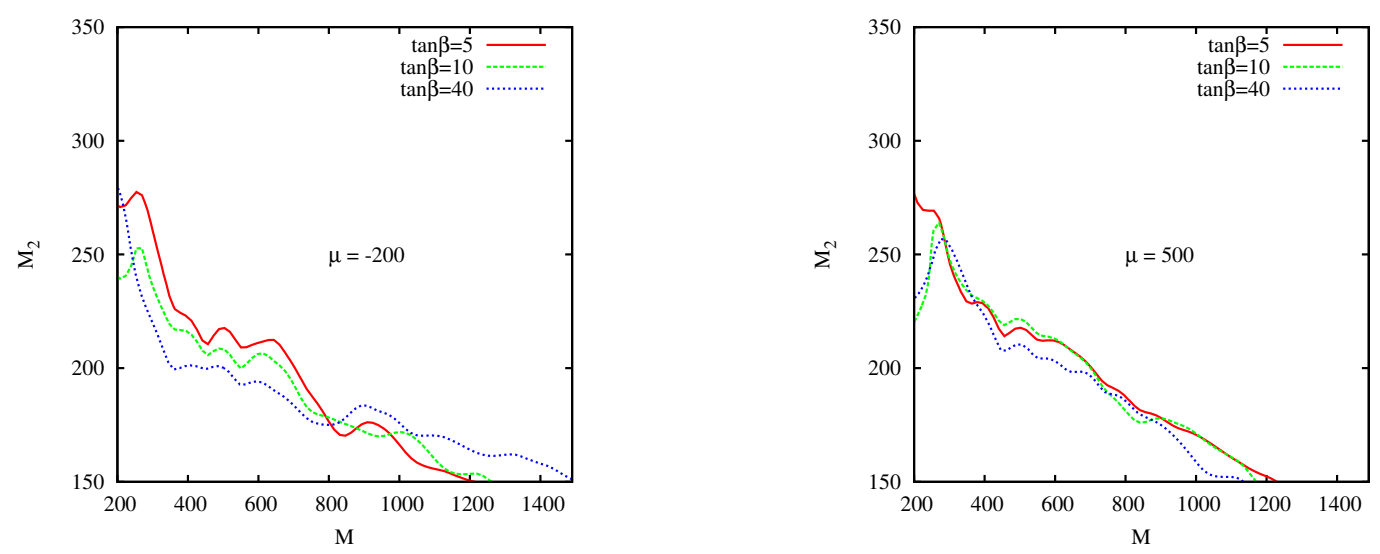

FIG. 6: Exclusion curves for Case-C (sbottom lightest scenario). The x-axis refers to the mass of $\tilde{b}_{1}$ squark.

The dependence of the exclusion curves on $\tan \beta$ is shown in Fig. 5, For the case of $\mu=-200$ $\mathrm{GeV}$ and $\tan \beta=5$, we find that $\tilde{t}_{1}$ and $\tilde{b}_{1}$ up to $300 \mathrm{GeV}$ are ruled out for gluino masses up to $700 \mathrm{GeV}$. The region just below the $\tilde{t}_{1}$ or $\tilde{b}_{1}$-LSP region is still allowed, as the near-degeneracy of their mass and the mass of the LSP results in a low missing energy and $M_{\text {eff }}$ spectrum which does not satisfy the hardness cuts imposed.

\section{Results: Case C}

This case considers the situation where both the stop states are heavier than the lightest sbottom state. The primary production processes are $\tilde{g} \tilde{g}$ and $\tilde{b}_{1} \tilde{b}_{1}^{*}$. As before, we disallow the region 

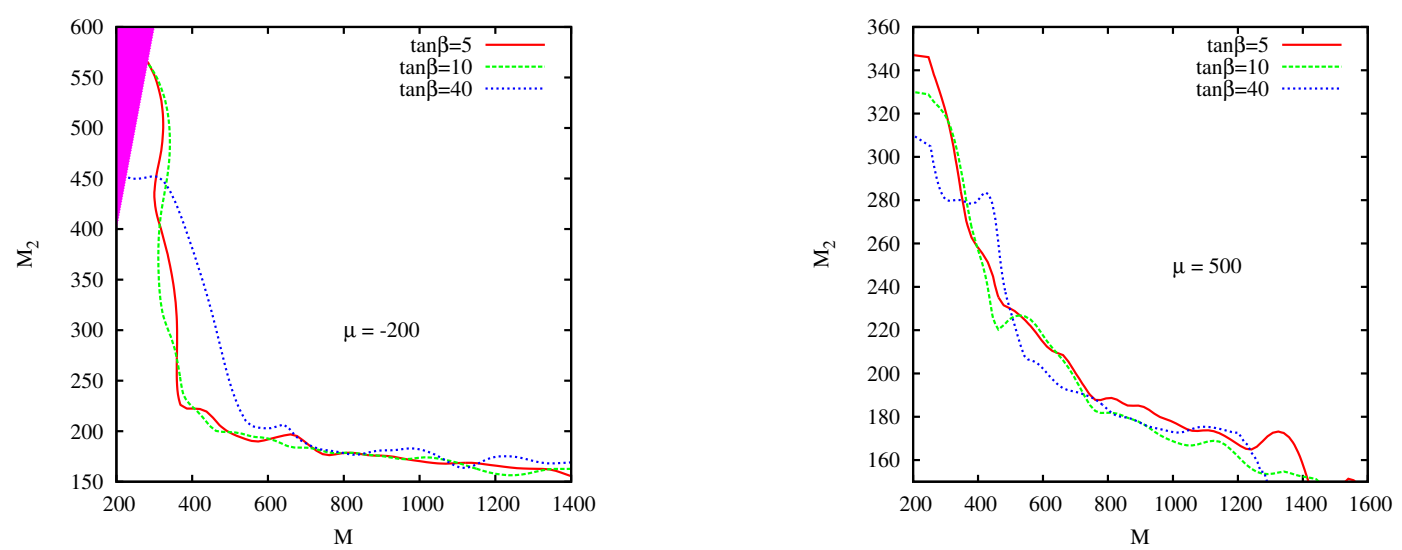

FIG. 7: Exclusion curves for Case-D (maximal mixing scenario). The $\mathrm{x}$-axis refers to the common mass of all the third generation squarks. The shaded region at the top corresponds to stop LSP and is therefore ruled out.

$M_{\tilde{b}_{1}}<M_{\tilde{\chi}_{1}^{0}}$. The region $M_{\tilde{\chi}_{1}^{0}}<M_{\tilde{b}_{1}}<M_{\tilde{\chi}_{1}^{+}}$corresponds to a $\tilde{b}_{1}$-NLSP with the dominant decay $\tilde{b}_{1} \rightarrow b \tilde{\chi}_{1}^{0}$. The gluino dominantly decays via $b \bar{b} \tilde{\chi}_{1}^{0}$ in the region $M_{\tilde{g}}<M_{\tilde{b}_{1}}$ and to $b \tilde{b}_{1}$ otherwise. This case is the closest to the scenarios considered by the ATLAS collaboration for the interpretation of their b-jet and missing energy searches. They split their analysis into a case where they disallow any three body decays of the gluino via $\tilde{g} \rightarrow b \bar{b} \tilde{\chi}_{1}^{0}$ and the case of a simplified model where there are no two-body decays but all decays are via this channel. According to the first case, they rule out gluino masses up to $720 \mathrm{GeV}$ for $\tilde{b}_{1}$ masses up to $600 \mathrm{GeV}$ [16]. As can be seen from Fig. 6 , for $\tilde{b}_{1}=600 \mathrm{GeV}$, we rule out $M_{\tilde{g}}<570-660 \mathrm{GeV}$ for $\mu=-200$ and $M_{\tilde{g}}<$ $600-660 \mathrm{GeV}$ for $\mu=500 \mathrm{GeV}$.

\section{Results: Case D}

In the maximal mixing scenario in both the stop and sbottom sector, all four squarks of the third generation have nearly degenerate masses. Therefore the production cross section is maximum for this scenario and the limits are strongest. The decay scheme for the sbottom is same as in Case B whereas for the stop, it is the same as in Case A. The gluino can now decay both via stops or sbottoms, but the large mass of the top means it decays preferentially via $b \tilde{b}_{i}$ channels. As expected, low $\mu$ results in large exclusion in $M_{2}$ for low squark masses. The dependence of the limits on $\tan \beta$ is shown in Fig. 7. The case for $\mu=-200 \mathrm{GeV}$ (and similarly $\mu=200 \mathrm{GeV}$ ) result in an exclusion of third generation squark masses of $280 \mathrm{GeV}$ for all allowed values of $M_{2}$. 
This case is similar to the one considered in [35], where, for naturalness requirements they require $\tilde{t}_{R}, \tilde{t}_{L}$ and $\tilde{b}_{L}$ to be degenerate and assume a Higgsino LSP. Their limits on the mass of the third generation squarks lie between 200-300 GeV.

The exclusion limits for the case of $\mu=500 \mathrm{GeV}$ does not show any exclusions that are independent of gaugino (and hence gluino) masses. The third-generation squark masses are completely un-constrained for $M_{\tilde{g}}>1 \mathrm{TeV}$. Whereas, the approximate requirement for naturalness that $M_{\tilde{t}_{1}}<500 \mathrm{GeV}$ would translate into $M_{\tilde{g}}>600 \mathrm{GeV}$.

In conclusion, among the four cases discussed above, only the case with degenerate thirdgeneration squarks and low- $\mu$ leads to mass limits independent of gluino mass - that of $280 \mathrm{GeV}$. In most other cases, we find that limits depend strongly on the composition of the neutralinos and charginos. The case where only $\tilde{t}_{1}$ is accessible is the least constraining, mainly due to low production cross sections compared to the other cases. For the case where the LSP is a almost pure Bino (high- $\mu$ ), $M_{\tilde{t}_{1}}=200 \mathrm{GeV}$ is ruled out for a gluino mass less than $570 \mathrm{GeV}$. Taking into account all values of $\mu$ and $\tan \beta, M_{\tilde{t}_{1}}=200 \mathrm{GeV}$ is ruled out for gluino masses in the range $570-720 \mathrm{GeV}$.

The case of lightest third generation squark being $\tilde{b}_{1}$ is the most insensitive to variations of both $\mu$ and $\tan \beta$. For this case, our limits are consistent with ATLAS's own interpretations within $10 \%$. $M_{\tilde{b}_{1}}=200 \mathrm{GeV}$ is ruled out for gluino masses between $680-820 \mathrm{GeV}$. The case of degenerate lefthanded squarks rules out $M_{\tilde{t}_{1}}=M_{\tilde{b}_{1}}=200 \mathrm{GeV}$ for gluino masses in the range $900-1050 \mathrm{GeV}$. And finally, the case with all squarks degenerate rules out $M_{\tilde{t}, \tilde{b}}=200$ for $M_{\tilde{g}}<900 \mathrm{GeV}$ in the worst case, and for all gluino masses in the best case.

\section{HIGH-SCALE NON-UNIVERSAL SCALAR SCENARIOS}

Besides the low-scale study done in the previous section, it is also possible to perform a scan over high-scale parameters. The advantage of a high-scale analysis is that the hierarchy of the particles is uniquely and consistently determined from the renormalisation group (RG) running of masses to low scale from given high-scale parameters. We use the simplification afforded by this model to include the effects of sleptonic sector in our analysis.

It is possible that the limits described in the previous section are diluted if slepton masses are allowed to be light. This is because the gauginos would then decay predominantly to sleptons resulting in leptonic final states which would be discarded since all the analyses considered here 
have a lepton veto. Including the full lepton sector in the low-scale pMSSM requires five more parameters and makes a general study far more complicated. We therefore leave a fully model independent investigation of interpreting the ATLAS limits involving a low mass slepton sector to a future work. However, we partially answer the question as to whether the limits are diluted by studying some illustrative cases, as described below.

Even though the soft-scalar masses may in principle take separate values, the constrains from flavour changing neutral currents (FCNC) from meson decays dictate that the first two generation squarks remain degenerate. Similarly, absence of decays like $\mu \rightarrow \gamma e$ means that the masses of first two generations of sleptons also have to degenerate. Therefore we can consider three schemes of non-universality:

- Case HA: Third generation squarks are lighter than all other sfermions. This will lead to a hierarchy similar to Case $\mathrm{A}$ in the preceding analysis.

- Case HB: Third generation squarks and sleptons are lighter than all other sfermions. This leads to light staus and tau-sneutrinos. Possibly, this would lead to $b \tau$ final states which have been studied in [51].

- Case HC: Third generation squarks and all sleptons are light. Comparing the limits in this case to those in Case HA will answer the question of dilution of limits due to leptonic signatures.

As in the previous analysis, we retain the cMSSM-like gaugino masses. We now have two mass scales in the scalar sector - the scale of heavy, decoupled particles $\left(M_{\text {heavy }}=2 \mathrm{TeV}\right)$ and the scale of light scalars $\left(M_{\text {light }}\right)$. We once again assume that the gaugino sector follows the universal structure and we set the higgs mass parameters for the two higgs doublets to be same as $M_{\text {light }}$. The parameters are all set at the grand unified theory (GUT) scale and the TeV-scale values are determined by RG running using the program SUSPect. The exclusion curves obtained for the three cases are shown in Fig. 8 for a value of $\tan \beta=10$. The results for values of $\tan \beta=$ 5, 40 are similar. We do not see any significant difference among the exclusion curves for the various high-scale cases. This can be interpreted as the robustness of the zero-lepton signals against different slepton masses and justifies the assumption of decoupled slepton masses made in the previous section. 


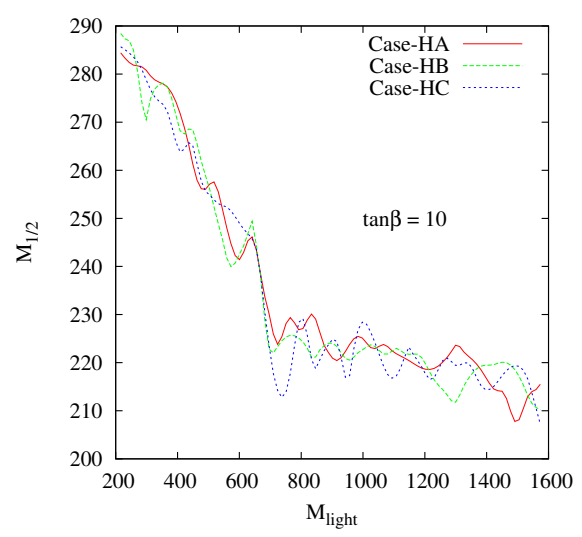

FIG. 8: Comparison of exclusion curves from three high-scale non-universal scenarios. All cases agree with each other within statistical uncertainties.

\section{CONCLUSIONS}

We have investigated the consequences of the recent ATLAS data in channels with (b-)jets and missing energy on the limits on the mass of the third generation squarks. We work in the pMSSM framework, with $\mathrm{TeV}$-scale parameters, without requiring a high-scale breaking scheme. For obtaining relatively model independent limits on the third generation squark masses, we decouple the first two squark generations as well as all sleptons. We also explicitly show that decoupling of sleptons is not likely to affect the limits as long as we work with 0-lepton signatures. We find that a stop of mass $200 \mathrm{GeV}$ can be ruled out for a gluino mass of $570 \mathrm{GeV}$ in the least constraining case whereas a stop of mass $500 \mathrm{GeV}$ is allowed for gluino masses upward of 450-880 GeV depending on the structure of the third-generation squark sector, and the parameters $\mu$ and $\tan \beta$. In the case where all third generation squarks are degenerate, we can rule out masses less than $280 \mathrm{GeV}$ for $|\mu| \leq 200 \mathrm{GeV}$, independent of the gluino mass.

One may also question the assumption of the gaugino mass pattern of $M_{1}: M_{2}: M_{3} \simeq 1: 2: 6$. We can expect the case of right-handed sbottom being the lightest of the third generation to be fairly independent of the assumption of gaugino mass pattern since the only dominant decays are $\tilde{b}_{1} \rightarrow b \tilde{\chi}_{1}^{0}$ and $\tilde{g} \rightarrow b \bar{b} \tilde{\chi}_{1}^{0}$, and both are always allowed (except for very compressed spectra). We would expect significant deviations from the stop limits when, for example, the decay $\tilde{t}_{1} \rightarrow b \tilde{\chi}_{1}^{+}$ is largely inaccessible because both $M_{2}$ and $\mu$ are so high that the charginos are generally heavier than the stop. In this case, the dominant decay for most of the parameter space would be $\tilde{t}_{1} \rightarrow t \tilde{\chi}_{1}^{0}$. In the current study, regions where the $t \tilde{\chi}_{1}^{0}$ decay was kinematically disallowed was still largely 
covered by the $b \tilde{\chi}_{1}^{+}$decay, thus leaving only a small region of parameter space corresponding to the flavour violating decay. However, in the absence of the decay into a chargino, one would need to examine in detail, the relative strengths of the (highly model-dependent) flavour-violating decay $\tilde{t}_{1} \rightarrow c \tilde{\chi}_{1}^{0}$ and the three-body decay of the stop. In other cases, where the stop still decays via standard channels, we do not expect significant deviations from our limits.

Acknowledgements: We thank Bruce Mellado for information on smearing parameters for detector effects. This work was partially supported by funding available from the Department of Atomic Energy, Government of India for the Regional Centre for Accelerator-based Particle Physics, Harish-Chandra Research Institute. Computational work for this study was partially carried out at the cluster computing facility of Harish-Chandra Research Institute (http://cluster.mri.ernet.in).

[1] H. P. Nilles, Phys. Rept. 110, 1 (1984).

[2] S. P. Martin (1997), hep-ph/9709356.

[3] A. H. Chamseddine, R. L. Arnowitt, and P. Nath, Phys.Rev.Lett. 49, 970 (1982).

[4] R. L. Arnowitt and P. Nath, Phys.Rev.Lett. 69, 725 (1992).

[5] G. L. Kane, C. F. Kolda, L. Roszkowski, and J. D. Wells, Phys.Rev. D49, 6173 (1994), hep$\mathrm{ph} / 9312272$.

[6] G. Giudice and R. Rattazzi, Phys.Rept. 322, 419 (1999), hep-ph/9801271.

[7] C. F. Berger, J. S. Gainer, J. L. Hewett, and T. G. Rizzo, JHEP 0902, 023 (2009), 0812.0980.

[8] G. Aad et al. (The ATLAS Collaboration) (2009), 0901.0512.

[9] P. Brax and C. A. Savoy, JHEP 0007, 048 (2000), hep-ph/0004133.

[10] S. Dimopoulos, G. Giudice, and N. Tetradis, Nucl.Phys. B454, 59 (1995), hep-ph/9504296.

[11] G. Aad et al. (Atlas Collaboration) (2011), 1102.5290.

[12] G. Aad et al. (Atlas Collaboration), Phys.Rev.Lett. 106, 131802 (2011), 1102.2357.

[13] G. Aad et al. (ATLAS) (2011), 1103.4344.

[14] G. Aad et al. (ATLAS) (2011), aTLAS-CONF-2011-086.

[15] G. Aad et al. (ATLAS) (2011), 1109.6572.

[16] G. Aad et al. (ATLAS) (2011), aTLAS-CONF-2011-098.

[17] K. Sakurai and K. Takayama (2011), 1106.3794. 
[18] K.-i. Hikasa and M. Kobayashi, Phys.Rev. D36, 724 (1987).

[19] C. Boehm, A. Djouadi, and Y. Mambrini, Phys.Rev. D61, 095006 (2000), hep-ph/9907428.

[20] M. Muhlleitner and E. Popenda, JHEP 1104, 095 (2011), 1102.5712.

[21] R. Kadala, P. Mercadante, J. Mizukoshi, and X. Tata, Eur.Phys.J. C56, 511 (2008), 0803.0001.

[22] M. Graesser and J. Shelton, JHEP 0906, 039 (2009), 0811.4445.

[23] N. Bhattacharyya, A. Datta, and M. Maity, Phys.Lett. B669, 311 (2008), 0807.0994.

[24] N. Desai and B. Mukhopadhyaya, Phys.Rev. D80, 055019 (2009), 0901.4883.

[25] W. Beenakker, S. Brensing, M. Kramer, A. Kulesza, E. Laenen, et al., JHEP 1008, 098 (2010), 1006.4771 .

[26] H. Baer, S. Kraml, A. Lessa, S. Sekmen, and X. Tata, JHEP 1010, 018 (2010), 1007.3897.

[27] A. Bartl, H. Eberl, B. Herrmann, K. Hidaka, W. Majerotto, et al., Phys.Lett. B698, 380 (2011), 1007.5483.

[28] H. Li, W. Parker, Z. Si, and S. Su, Eur.Phys.J. C71, 1584 (2011), 1009.6042.

[29] S. Bornhauser, M. Drees, S. Grab, and J. Kim, Phys.Rev. D83, 035008 (2011), 1011.5508.

[30] K. Huitu, L. Leinonen, and J. Laamanen (2011), 1107.2128.

[31] A. Datta and S. Niyogi (2011), * Temporary entry *, 1111.0200.

[32] Y. Kats, P. Meade, M. Reece, and D. Shih (2011), 1110.6444.

[33] R. Essig, E. Izaguirre, J. Kaplan, and J. G. Wacker (2011), 1110.6443.

[34] X.-J. Bi, Q.-S. Yan, and P.-F. Yin (2011), 1111.2250.

[35] M. Papucci, J. T. Ruderman, and A. Weiler (2011), 1110.6926.

[36] C. Brust, A. Katz, S. Lawrence, and R. Sundrum (2011), 1110.6670.

[37] T. Sjostrand, S. Mrenna, and P. Z. Skands, JHEP 0605, 026 (2006), hep-ph/0603175.

[38] W. Beenakker, R. Hopker, and M. Spira (1996), hep-ph/9611232.

[39] M. Cacciari, G. P. Salam, and G. Soyez, JHEP 04, 063 (2008), 0802.1189.

[40] A. Djouadi, J.-L. Kneur, and G. Moultaka, Comput.Phys.Commun. 176, 426 (2007), hep-ph/0211331.

[41] J. A. Conley, J. S. Gainer, J. L. Hewett, M. P. Le, and T. G. Rizzo, Physical Review D (2011), 1103.1697.

[42] S. Sekmen et al. (2011), 1109.5119.

[43] B. Allanach, Phys.Rev. D83, 095019 (2011), 1102.3149.

[44] B. Allanach, T. Khoo, C. Lester, and S. Williams, JHEP 1106, 035 (2011), * Temporary entry *, 1103.0969. 
[45] O. Buchmueller, R. Cavanaugh, A. De Roeck, M. Dolan, J. Ellis, et al. (2011), * Temporary entry *, 1110.3568 .

[46] D. Feldman, K. Freese, P. Nath, B. D. Nelson, and G. Peim, Phys.Rev. D84, 015007 (2011), 1102.2548 .

[47] P. Athron, S. King, D. Miller, S. Moretti, and R. Nevzorov, Phys.Rev. D84, 055006 (2011), 1102.4363.

[48] S. Scopel, S. Choi, N. Fornengo, and A. Bottino, Phys.Rev. D83, 095016 (2011), 1102.4033.

[49] S. Akula, N. Chen, D. Feldman, M. Liu, Z. Liu, et al., Phys.Lett. B699, 377 (2011), * Temporary entry *, 1103.1197 .

[50] T. Aaltonen et al. (CDF) (2009), search for scalar top decaying into $c+\tilde{\chi}_{1}^{0}$ in the MET+jets sample; CDF Note 9834.

[51] N. Bhattacharyya, A. Choudhury, and A. Datta (2011), 1107.1997.

[52] B. Mellado, private communication. 